\title{
Application of BP Neural Network in Face Recognition
}

\author{
Wen.Yang Yu \\ Information Engineering College \\ Ningbo Dahongying College \\ Ningbo, People's Republic of China \\ seayuweya@163.com
}

\author{
Xian.Wei WU, Yu.Bin Yang \\ Information Engineering College \\ Ningbo Dahongying College \\ Ningbo, People's Republic of China \\ wxw786@qq.com
}

\begin{abstract}
A face recognition model is established, and three training strategies i.e. sampling training, batch training and integrity training are studied. The recognition model designed includes image compression, image sampling, input vector standardization, BP neural network and competition selection. Experimental results demonstrate that the recognition model is simple and has a high recognition rate.
\end{abstract}

Keywords- BP Network; Neural network; Image compression; Pattern recognition; Face recognition

\section{INTRODUCTION}

Face towards identifying major rise in two areas (1) the widespread use of the camera creates a demand for people in front of computers automatic shooting. By using color and size of the operation, can easily locate the person sitting in front of the computer face. By determining that the person is staring straight face to have a computer or deflection can know whether the current user interface attractive to users, which in children's education systems, computer games, and even traffic electronic eyes have a wide range of applications. (2) Used in video retrieval or content-based image retrieval precise person's face toward the system. Traditional detection methods for each face towards the corresponding detector are designed, while the system may face toward the face direction corresponding to various suitable detectors which can reduce the error rate and adjusting the computational cost of each detector. In summary, the identification of the face toward the video recognition analysis of human behavior is significant, toward the free face is typical of an important part of the discriminant analysis problems. The subject of this study is based on the picture of the face towards the classification problem, by a group of people face image analysis, image library five images per person, face orientation were: left, front left, front, right front, and the right, shown in Figure 1:

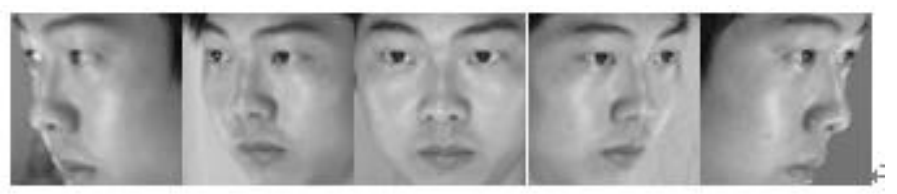

Figure 1. Face orientation: left, front left, front, right front, and the right.

Different characteristics of the person towards the parts, such as the position of the eyes, nose, and mouth clear differences between the orientations of this as the basis for identification.

\section{BP NEURAL NETWORK FACE TOWARD RECOGNITION}

BP network is based on the error back propagation algorithm (Error Back Propagation) to construct the multi-forward neural network, which is before the nonlinear differentiable function to weight training multilayer networks. Currently BP network has become the most widely used neural network, according to statistics; nearly $90 \%$ of the neural network is based on the BP network.

Structurally, the most left-layer BP network input layer, the right-most layer is the output layer, the middle of the hidden layer, which layers may be one or more layers (as shown), each layer of the network connection weights can be adjusted by learning. BP network consists of an input layer, an intermediate layer and an output layer, the intermediate layer is hidden layer may be a layer or layers. In this system, uses a three-layer BP network, which is only one hidden layer. 


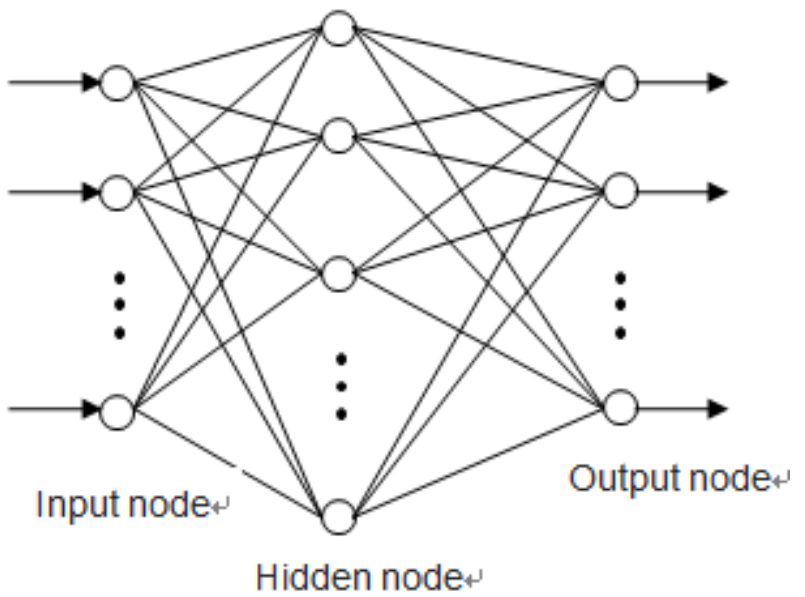

Figure 2. Three-layer BP network.

\section{A. Design input layer and output layer}

The number of input layer neurons depends on the input dimension of the sample. If a face image of size $420 \times 420$ pixel gray feature direct input, the input layer node number will reach 176,400 , the general practice is to reduce the dimension of the input image and extract effective features. Therefore, the number of nodes in the input layer depends on the number of sample characteristics. Use BP network as a classifier, the number of human face toward its category number (i.e., left, left front, front, right front and right) classification, so the number of nodes in the output layer is 3.3 nodes can take some of this combined value: 110,100,010,001,011, described here in order to facilitate the issue, let us first briefly consider the output is an integer, each node in the output layer that is a non-zero value, when the three nodes to 0 , returns rejection.

\section{B. Select the hidden layer nodes}

Hidden nodes choice is very complex, and if the number of nodes is too small, the network will not be able to create complex judgments boundary, so that the network cannot be trained, or even trained, the network is not "strong", not previously see if they cannot identify over the sample, fault tolerance is poor; But if there are too many, it will make the learning time is too long, slow convergence require a longer training time, generalization ability of the network at the same time will be reduced, but the error is not necessarily the best, hence the need to find an optimal hidden nodes.
If a single training three BP network hidden layer, the first hidden layer can initially choose the number of nodes according to the following empirical formula:

$$
h=\sqrt{n+m}+a
$$

Where, $\mathrm{m}$ is the number of input nodes, $\mathrm{n}$ is the number of output nodes, usually, a value that can be an integer from 1 to 10 in less than, the specific values of hidden nodes or to be the case based on the experimental results good or bad decision. Because the ability to consider the correct classification of the network, but also consider the network training convergence speed and other factors in order to better judge.

How to select the number of hidden layer neurons is appropriate, it does not have a clear theoretical conclusions, literature mentioned are: trial and error method, growth method, pruning methods, evolution and adaptive methods.

The empirical formula is used:

$$
S_{\max }=\operatorname{int}\left[\operatorname{sqrt}\left(\begin{array}{l}
0.43 m n+0.12 n^{2}+ \\
0.54 m+0.77 n+0.86
\end{array}\right)\right]
$$

Here, int $(\mathrm{x})$ is a rounding function, $\mathrm{m}$ and $\mathrm{n}$ represents the number of input layer and output layer neurons.

\section{EXPERIMENTS AND RESULTS ANALYSIS}

\section{A. Face image preprocessing}

\section{1) Image Compression}

The input image is generally relatively high accuracy; there is a lot of redundant information. To avoid neural network structure is too complex, before entering the neural network must be image compression. Image compression usually interpolation algorithms, including neighbor interpolation, bilinear interpolation and bicubic. Interpolation algorithm to the image quality of the relationship between the degree of distortion, interpolation function is designed to be the core issue of the interpolation algorithm.

A represents $f(x)$ set interpolation, $h(x)$ represents the interpolation kernel, the interpolation formula for

$$
f(x)=\sum_{i=0}^{K-1} C_{i} h\left(x-x_{i}\right)
$$

Where Ci represents the weight factor, The formation of different options interpolation nuclear different interpo- 
lation algorithms. This article cubic interpolation kernel function, the size of the interpolation neighborhood chosen $4 \times 4$, then the size of the output pixel value is the weighted average effective point $4 \times 4$ matrix contains.

\section{2) Image sampling}

The so-called image sampling is the two-dimensional image matrix compression line after line after pulling into a one-dimensional column vector, and its purpose is to facilitate the subsequent neural network input.

\section{3) Input vector standardization}

Image sample output is one-dimensional column vector whose elements range is $[0,255]$. If you direct the column vector input neural network training, due to the greater value, is bound to affect the calculation of the efficiency and convergence rate. Therefore, before the column vector input neural network input vector standardization should be implemented.

Set of input vectors for $\mathrm{x} \in \mathrm{R}^{\mathrm{d}}$, output vector for $\mathrm{x}^{\prime} \in \mathrm{R}^{\mathrm{d}}$, $\mathrm{X}_{\max }$ is the maximum value of the input vector $\mathrm{x}, \mathrm{x}_{\min }$ is the input vector $\mathrm{x}$ in the minimum, then the input vector standardization process can be described as:

$$
\mathrm{x}^{\prime}=\frac{\mathrm{x}-\mathrm{x}_{\min }}{}
$$

Obviously, the input vector standardization is implemented linear mapping of the input vector, the input vector elements range from [0,255] convert between $[0,1]$ easier to adjust and subsequent operation of neural network weights and thresholds.

\section{B. Training programs}

In this paper, the original image is taken from the ORL face database. The database consists of 40 people, each 10 , the size of $112 \times 92$, gray scale face image 256 formed. Training set consists of a total of five former 200 per person, the face image is composed, the test set by the person 5 Total 200 face images composed, and the training set and test set of face images do not overlap. Sample arrangement of the training set for five sample image of the first category; 5 image of the second category of samples; ...; 5 40th class image samples.

After the input image compression processing, converted the $112 \times 92$ to $7 \times 6$ dimensional image dimension. BP network input layer nodes 42, 70 hidden layer nodes and output layer nodes are 40. Experiment, with the use of the three training strategy, the formation of several training programs are as follows.
Scheme I. First sampled the implementation of the program of training samples, and then complete the implementation of the training sample. 5 is taken as the sampling interval. That first image of the first one first class training samples, and then a picture of the first second category of samples for training, and so on, until the first one image 40 categories of training samples have been exhausted. Finally, the sample integrity training.

Scheme II. To implement the odd sample sampling training, followed by the implementation of the even samples sampling train, the last sample of the full implementation of the training.

Scheme III. Sample batch training program implementation. That is the first of five first class image samples for training, then on the first category, 10 images a second category of samples for training, and then the first category, the first two categories, the first three categories of samples 15 images training, and so on, until the completion of training for 200 samples so far.

Scheme IV First sample batch program to implement the training, and then complete the implementation of the training sample. The samples of the same class in the training set during the training a batch training, i.e., five images of the same category as a group for training.

Scheme $V$ Implemented a complete training program samples.

The difference scheme I and II of the program is that the sampling interval. Every program I sample are different categories of income, the larger the sampling interval. III differs from program to program IV that the batch size. III program by batch size is the number of samples of different types of exponentially growing; while program IV batch size is fixed on the number of samples of the same class.

Table1 Compares the various training programs for face recognition when 1 BP Network

\begin{tabular}{|c|c|c|c|c|}
\hline $\begin{array}{c}\text { Training } \\
\text { programs }\end{array}$ & $\begin{array}{c}\text { FAR } \\
\text { training } \\
\text { set\% }\end{array}$ & $\begin{array}{c}\text { FRR } \\
\text { training } \\
\text { set\% }\end{array}$ & $\begin{array}{c}\text { FAR test } \\
\text { set \% }\end{array}$ & $\begin{array}{c}\text { FRR test } \\
\text { set\% }\end{array}$ \\
\hline Scheme I & 0 & 0 & 6.5 & 0 \\
\hline Scheme II & 0 & 0 & 7.5 & 0 \\
\hline Scheme III & 0 & 0 & 9.5 & 0 \\
\hline Scheme IV & 0 & 0 & 7.5 & 0 \\
\hline Scheme V & 0 & 0 & 10.5 & 0 \\
\hline
\end{tabular}


Table 1 shows the experimental results. Where the sample sampling error and sample batch training error are taken as 0.62 , the sample is taken as 0.5 full training errors, the maximum limit of 876 training times, learning rate to take 0.01 .

From Table 1, the training program I recognition rate 93.5\%; program II and IV programs reached $92.5 \%$, up 90.5 percent III program, the program V up to $89.5 \%$. All programs of training and test sets of FRR were $0 \%$, the training set of learning was $100 \%$. Obviously, I plan to identify higher than plan II, described BP network continues to study samples of the new category, better network performance. IV identification scheme is higher than the scheme III, described BP networks will all samples of the same class together to learn, better network performance. Scenario III recognition rate is not very high, but it has a feature that when increasing the number of training samples, the entire network without having to re-train, just to add to the training samples. $\mathrm{V}$ program lowest recognition rate, indicating that the training sample gradually added more than a one-time adjustment to join the network performance is better. Conventional training methods commonly used scheme $\mathrm{V}$, sample integrity training strategy if you plan to use $\mathrm{V}$ compatible with other training strategies, such as the scheme I, the recognition rate will be greatly enhanced.

In this paper the literature [1] neural network error rate of $3.8 \%$ compared to the program, although I error rate was $6.5 \%$, but the recognition model is simple and can meet the actual requirements. And training strategy program I use to make BP network has good nonlinear mapping feature, reached a high recognition rate. This also shows that BP network to simulate the working mechanism of the human brain, as people in the learning process if appropriate learning method can improve the efficiency of learning the same.

\section{CONCLUSION}

This article will BP neural network for face recognition, the establishment of a recognition model to study the sam- ple sampling train, the impact of training and sample the complete sample batch training three training strategies for recognition rate. Simulation experiments with ORL face database, the results show that the simple recognition model in this paper, the use of proper training strategy to improve the training speed and training efficiency, making the model classification performance has improved significantly. Therefore, the identification model of this article and training strategy is feasible in practical applications. BP network can be the root cause of face recognition is that it can achieve a special nonlinear mapping the input space is transformed to the output of the sheets into the space, making the output space classification problem becomes simple.

\section{REFERENCES}

[1] L.M Zhang , N Liu, P.Y Yu, A Novel Instantaneous Frequency Algorithm and Its Application in Stock Index Movement Prediction, 2012. Selected Topics in Signal Processing: IEEE Journal of Volume: 6, Issue: 4,pp. 311-318

[2] Liumei Zhang, Jianfeng Ma, Yichuan Wang and Shaowei Pan, "PSO-BP Neural Network in Reservoir Parameter Dynamic Prediction," Computational Intelligence and Security, 2011,pp. 123 - 126,DOI: 10.1109/CIS.2011.35

[3] Z.B Xu ,R Zhang,W.F Jing, When Does Online BP Training Converge?, Neural Networks, 2009. IEEE Transactions, pp.1529-1539

[4] Chen Shihong and Liu Xiaoqing " Research of Population Prediction Based on GA-BP Neural Network," Computer Science \& Service System, 2012,pp.1193 - 1196, DOI: 10.1109/CSSS.2012.302

[5] R Zhang,Z.B Xu,G.B Huang,D.H Wang, Global Convergence of Online BP Training With Dynamic Learning Rate, Neural Networks and Learning Systems, IEEE Transactions, 2012,IEEE Journals \& Magazines,pp. 330-341

[6] Kewen Liu , Haiming Zhou, Zhanyong Yang and Fumin Qu, "Application of BP Neural Network for Line Losses Calculation Based on Quantum Genetic Algorithm," Computational Intelligence and Design, 2011,pp. 3 - 7, DOI: 10.1109/ISCID. 2011.9

[7] S.J Zhai,T Jiang, Sparse representation-based feature extraction combined with support vector machine for sense-through- foliage target detection and recognition, IET: Signal Processing, 2014,pp. 458-466

[8] Yuan Ning, Yufeng Liu and Qiang Ji, "Bayesian - BP Neural Network based Short-term Load Forecasting for power system," Advanced Computer Theory and Engineering, 2010,pp. V2-89 V2-93, DOI: 10.1109/ICACTE.2010.5579151

[9] D.S Guo, Y.N Zhang, Novel Recurrent Neural Network for Time-Varying Problems Solving, IEEE: Computational Intelligence Magazine, 2012, pp.61-65

[10] Xue-Feng Jiang, "The research on sales forecasting based on rapid BP neural network," Computer Science and Information Processing, 2012,pp. 1239 - 1241, DOI: 10.1109/CSIP. 2012.6309083 